\title{
Student Learning on Ethical Educational Placements
}

I grew in confidence and became a level headed professional, not just an awkward student. (Nurse, Uganda)

\section{TRANSFORMATIONAL LEARNING}

As with the findings from our study of Professional Voluntarism, ${ }^{1}$ it is difficult to isolate the different elements of learning experienced through placements since personal and professional skills overlap and some aspects of learning are almost intangible, particularly those transformational elements which characterise most students' accounts. The following two responses illustrate the transformational impacts as perceived by the students on placement:

I just feel like it's helped me change my outlook and personality. (Midwife, Uganda)

During my time there I went through a wave of different emotions from anger, frustration, passion, amazement and joy to name just a few ...After returning home and having more time to reflect I believe that the experience had changed me on a level I wasn't expecting. (Nurse, India)

Fee and Gray capture the gains in capabilities and the value of skills gained in low resource settings to contemporary workplaces. They conclude that

${ }^{1}$ See Chapter 3 in Ackers and Ackers-Johnson (2016).

(C) The Author(s) 2017 
such skills are not easily codified and taught: they are distinctively generic, rather than technical; tacit rather than explicit; higher order rather than basic; portable rather than profession or situation-bound and 'soft' (interpersonal) rather than 'hard' (technical):

Notably, expatriates experienced learning outcomes that were more frequently transformational, involving fundamental changes to their values, perspectives or assumptions. (2013: 196)

There is strong evidence to suggest that stays in low resource settings create opportunities for accelerated and complex learning and that the outcomes of these are precisely the kinds of competences needed to drive innovation and efficiency in an increasingly resource-constrained NHS (Norton and Marks-Moran 2014). The potential gains of international elective placements for students are well-documented (see Brookfield 1995; Elit et al. 2011; Murdoch-Eaton and Green 2011) and encompass in general terms, both professional and personal development and the acquisition of new knowledge in and of different contexts (Ackerman 2010). In more specific terms, these positive impacts for students are believed to include: the development of clinical skills in a new context; knowledge of and experience in different health systems; professional development; the development of generic skills, including organisational skills, communication, negotiation, self-evaluation, cultural competence (Jeffrey et al. 2011), compassion towards patients, awareness of resource use, confidence, goal setting, widening students' perspectives, independence, and personal growth; reflective questioning of both the challenges and assumptions of practices; greater understanding of different value systems; and 'social accountability' (Brookfield 1995; Elit et al. 2011) leading to a range of 'socially responsible' educational outcomes (Murdoch-Eaton and Green 2011). Figure 3.1 summarises the findings of existing research on learning outcomes associated with international placements in low resource settings.

These domains map directly onto the UK's NHS Knowledge and Skills framework or 'KSF'. 2 The KSF is focused on staff development and appraisal and designed, 'to make it easier for staff to identify the core skills that they need to do their job and their development needs'. As such it is a

${ }^{2}$ http://www.nhsemployers.org/SimplifiedKSF 


\section{Knowledge and Skills}

\subsection{Clinical (Academic) Knowledge}

Substantive knowledge of contexts and health systems

Resource awareness, management and efficiency

Knowledge of research, evaluation and audit methods

\subsection{Clinical and Technical Skills}

Exposure to a high volume of (usual) clinical cases and new (unusual) cases

Generic, soft, interpersonal skills

Organisational and administrative skills

2. Values

2.1 Personal

Compassion/care

\subsection{Social}

Ethics, morality, social responsibility, social accountability; global citizenship

Equality and diversity

Cultural competence

\section{Service improvement and Leadership}

Problem solving, innovation and project management

Team working including multi professional working

4. Communication (links to cultural competence and leadership)

Teaching /presentational skills

Negotiation

Language skills

5. Personal Growth and Professional Development

Confidence

Goal setting

Independence

Self-evaluation and reflective questioning

Fig. 3.1 Summary of learning outcomes. Source: This summary is drawn from the authors cited above complemented by Dowell and Merrylees (2009) NHS HEE Toolkit (Longstaff 2012)

useful benchmark against which to evaluate learning on international placements. The KSF focuses on six dimensions:

1. Communication

2. Personal and people development 
3. Health, safety and security ${ }^{3}$

4. Service Improvement

5. Quality

6. Equality and Diversity

The following sections present the survey and interview findings under the headings outlined in Fig. 3.1.

\section{KNOWLEDGe AND SKILlS}

Perhaps the most obvious and tangible perceived benefit associated with international placements concerns the acquisition of new knowledge and skills. Indeed, for many, this may be the 'make-or-break' component underlining any justification for embedding such placements within higher education curricula (and committing resource to them). Drawing on the distinction between explicit and tacit skills (above) we have distinguished two components to this. Firstly, we discuss the acquisition of new knowledge. This includes both clinical and technical skills directly linked to the students' curricula and future profession and more foundational areas of knowledge about contexts, systems and resource management. We then move on to discuss the more operational dimension facilitating translation of knowledge into useable skills.

\section{Systems THINKING}

One of the areas students commonly referred to in terms of new knowledge could be loosely described as systems thinking. Students working in a narrow pocket of a large complex organisation (such as the NHS) often find it difficult to step back and consider the system as a whole. Spending time in a low resource setting where systems are malfunctioning is so very tangible that it forces students to engage in systems thinking as a component of problemsolving (see below). And, in the process of this, and from their position outside of the NHS they are then able to reflect on the NHS as a system.

Knowledge of how other health systems operate (Murdoch-Eaton and Green 2011) and experiencing different clinical environments (Dowell

${ }^{3}$ This is the only area that does not map directly onto identified learning outcomes. However, this forms a key focus of placement organisation in the EEPs. 
and Merrylees 2009) are often cited amongst the motivations for students to undertake elective placements and also as potential outcomes of them. Of the EEP students surveyed, $57 \%$ believed their placement had a very strong impact and $29 \%$ believed that their placement had strong impact on their learning regarding systems thinking. Some students felt that the insight gained through being placed in an unfamiliar context was illuminating in terms of facilitating their understanding of healthcare systems and different ways of working to those in the UK:

It gave me a really good insight into health care systems abroad and different ways you know, the different methods that they use to do things. I thought it was really interesting to see how health care's developed ... and sort of the managerial aspects of the hospital 'cos it was run very differently to the way we run a hospital, so that was very interesting. (Nurse, India)

This student's experience while on placement also influenced her thinking about the status of the nursing profession in different cultural and clinical contexts. Through reflective questioning of the challenges and assumptions of practices she identified the seemingly paradoxical relationship between gender, nursing and status, where nurses in India were very highly trained and respected, yet were not able to utilise their clinical skills in practice:

I think nursing is viewed very differently over there to what it is here, and so I think they've got a lot of advancements to make in terms of autonomy and decision making. They [nurses] don't get the credit they deserve, self-esteem is very low. But that's very much a gender thing and a hierarchy sort of issue. Nursing is very low down, they have a lot more clinical skills than we are taught but they are not even allowed to dress wounds. (Nurse, India)

Other students commented on the way systems and practices operated in their placement organisation comparing this with the UK. Some went beyond simple learning to demonstrate 'epistemic humility' (Hanson et al. 2013), noting how the availability of resources and organisational hierarchies significantly influenced practice:

We didn't want to be too intrusive, and tell them that that this is how they should do things because a lot of the ways that we would have done things used resources they didn't have. It is quite difficult having to take a step back and having a lot of listening and watching and not stepping in. It was quite a hierarchical system there. (Prosthetics \& Orthotics, Uganda) 
Some students were able to reflect and apply the learning gained while on placement to a deeper understanding of nursing practice in the $\mathrm{UK}$, and were able to make improvements to their own ways of working on return. This highlights how reflective observation gained within one context can allow learning from concrete experiences to be applied to another:

I think about it all the time when I'm on placements [in the UK]. It's just so different; we're all patient centred, patient focused, whereas over there it's more like task focused, like get the job done. And I mean, don't get me wrong, I think a lot of nurses over here can get like that too, when your workload is so big you can be like: 'Oh, you know we need to get this done, this done and this done' and when you're busy you know it can be like that, but I think that's what it's taught me as well, try to take your time with your patient and just explain what you're doing and, you know just have a chat and things like that. (Nurse, India)

For some students, being in a different context and experiencing different ways of working and learning from healthcare professionals facilitated a more questioning mind-set on return:

I think we learnt more from them, definitely the really good nurses and doctors, more from them than they have learnt from us. They were showing us things, the procedures and such. There's not really much over there that we see over here, it's completely different... I think it is really useful to see that 'cos it makes you come back and question why you do things in a certain way. (Nurse, Uganda)

It is clear from the evaluation that simply being outside of the system you are accustomed to and have been weaned in generates a capacity for systems thinking both in the host setting but also in relation to the UK NHS. And this form of learning applied to some degree in both hands-on (EEP) and observational-only (India) placements. One component of this system concerns management of scarce resources.

\section{Resource Awareness}

Awareness of resource use and resourcing of healthcare is an important learning outcome of international placements (Brookfield 1995; Elit et al. 2011; Murdoch-Eaton and Green 2011). From the post-placement survey, $74 \%$ and $14 \%$ respectively believed their placement had a very strong 
or strong impact on their resource awareness. Many students expressed shock and surprise at the lack of resources in the host countries:

I'm going from having all of your staff, equipment, tools and everything and going a month without it and then going back to it - it is a real shock. (Mental Health nurse, Uganda)

We've got the resources whereas they don't. Sometimes they just wouldn't have the medication in stock and that's just the way it is. If it wasn't in stock and the family couldn't afford certain medications, they'd just go without it, so that was quite tough. (Nurse, Uganda)

Some students were able to make direct links between the lack of resources in their placement setting to (global) structural inequality, and how this constrains the agency of healthcare workers:

The main challenge I saw in every clinic, workshop and teaching syllabus is the lack of resources. I know this is due to the lack of money available to the hospital and very little can be done to change this in the present economic state. (Prosthetics/Orthotics, Uganda)

Through concrete experience of the constraints in Uganda, students identified the challenges faced by healthcare workers and demonstrated an awareness of how these impacted upon practice. This was particularly marked in disciplines like prosthetics which rely quite heavily on materials:

I have been able to experience first-hand the numerous challenges encountered in a low-resource healthcare setting. It is clear that the greatest issue here is access to materials and machinery. (Prosthetics \& Orthotics, Uganda)

In the following case, a nursing student describes the lack of consumables and equipment that are essential to the effective performance of even the most basic nursing in Uganda:

One of my biggest challenges was the lack of resources that they had. This included equipment, gloves, medication and fluids. The lack of resources was not really overcome as we just had to work with what we had. I was fortunate that I had brought my own stethoscope and sphygmomanometer from home so I took that with me during my placement. (Nurse, Uganda) 
Although, as in the case described above, the lack of resources made it hard to perform even the most basic of tasks, others indicated that being in a low resource setting made them very conscious of how wasteful practices in the UK often were, and through this concrete experience, tacit knowledge and subsequent reflection, they changed their way of thinking about practice in the UK. This level of resource awareness also developed on observational placements in India but without the intensity of personal frustration:

They don't have the advanced equipment we have... they need the same equipment like we do. Because, at the end of the day they are patients as well. They are people so it's quite sad that we have all this technology. We probably waste so much. And they don't waste anything; they use everything they have. If we used less we could send it over there or to Africa. Or, to other countries where they need them. (Nurse, India)

These comments are interesting as they highlight the risk that students may generalise their experiences to caricature wider Indian or Ugandan health systems as facing severe resource and technology constraints when of course both countries have private sector hospitals with state of the art technology. This underlines the importance of reflective practice and having experienced mentors in country and on their return to the UK to frame and challenge the students' immediate experiences and systems thinking. Also, perhaps to encourage students to consider the practical impacts of donations. Nevertheless, in the context of NHS financial crisis, their experiences foster an acute awareness of waste:

I look at practice in a different way. I'm very conscious of the waste we produce now and the sort of the practice that we have. We're a bit nonchalant about things so I think it's been really good in that respect because you're very mindful of what you're using and whether you definitely need that or not. (Nurse, India)

Another student echoes this sentiment:

I learnt that people in the NHS take things for granted. I learnt that even though they had limited resources they made the most of what they had and in the NHS they waste so much. (Nurse, India) 


\section{Clinical and Technical Skills}

Clinical knowledge and skills have been identified as key learning attributes during electives (Dowell and Merrylees 2009). Practically all of the students felt that they had acquired clinical and practice-based knowledge, or technical skills (Williams 2006; Williams and Balatz 2008). Of the EEP students surveyed; $28 \%, 26 \%$ and $32 \%$ respectively thought their placements had a very strong, strong or moderate impact on their skills and competence. In many cases they spoke of having access to more specialist skills in their field or at the margins of their field that they have difficulty accessing during placements in the UK.

As we explained in Chapter 1, the India placements were organised via HEE on an observation-only basis whereas students in Uganda had the opportunity to practice, under supervision. The learning outcomes (in terms of clinical skills) are quite different. Students undertaking hands-on clinical placements reported intense and accelerated learning (Norton and Marks-Moran 2014; Stephens 2015). The following example is typical:

It was an experience and a half, I loved it. We did so much. I did more in those four weeks than I did in my whole first year and learned more in those four weeks than my first year. (Nurse, Uganda)

In the next case the occupational therapy student was able to gain key paediatric experience during her Ugandan placement:

It was good to get some paediatric experience, that was amazing because I don't think I would be able to get that experience [in the UK] because when you go to do something like that they want previous experience so that broke a bit of a barrier. (Occupational Therapist, Uganda)

Respondents used the concepts of 'exposures' or 'spoking-out' to identify unique learning encounters that they would not have had the opportunity to experience in the UK. Adult nursing students had the opportunity to work with babies or gain exposure to maternity cases, for example, which they had not had access to at home:

I've never really done [observations] on babies so being able to monitor them and see how they care for them. (Nurse, Uganda) 
I gained some clinical skills even doing clinical observations. Also the maternity side of things, I would never have otherwise known. (Nurse, Uganda)

I had lots of personal achievements. I assisted in the delivery of twins and 3 little girls. I took part in cervical screening which was an amazing experience and I was part of the hand hygiene training at Bukuuku health centre. (Nurse, Uganda)

Our ethnographic experience of working alongside students on placement, backed up by their interviews, suggests that placements in the UK are often quite narrowly defined in line with the specialisms they are working towards and students have limited opportunity to see beyond these and experience work at the interface of professional boundaries. This does not imply that the skills they gain are irrelevant. In some cases, students explained how the experience had influenced their future career ambitions perhaps involving a shift in emphasis. In other examples students referred to the value of this more holistic learning when they came to have placements in accident and emergency contexts (for example). In such environments, an adult nurse may well be faced with a pregnant mother or a child. Paramedic students who also spent time in health centres dealing primarily with maternity cases and children spoke of the value of this more multi-disciplinary learning to their future roles and the confidence it had given them to manage such cases. The broader exposure to cases that a student may not have immediate access to in the UK was also mentioned by mental-health nurses in Uganda who worked both at the very hard end of institutionalised and highly medicated patients and in community out-reach work:

In terms of mental health specifically, I've learned more about variation in diagnosis and how it can manifest and present differently. My placements so far [in the UK] had been quite stable and I just had to discharge patients, but there I could see crisis situations, which was quite an eye-opener. Eventually I will have to assess people in these conditions so it's given me knowledge of that. (Paramedic, Uganda)

In addition to this 'spoking out' most students in the Uganda placements referred to the level of access they had to more complex and emergency cases in their area of specialism. This was often related in their interviews 
to the issue of responsibility and confidence as the knowledge they had learnt from lectures and textbooks could now be applied in practice:

It has been invaluable to gain such varied clinical exposure and to have been given a level of access and responsibility that is beyond anything we have experienced in the UK... The hands-on exposure has allowed us to build upon our practical skills through assessing and providing patients with relevant treatments. (Prosthetics \& Orthotics, Uganda)

The same comments would doubtless be found amongst students who had taken part in traditional unsupervised electives and we are acutely aware of the line here between giving students access to cases that whilst presenting unique learning opportunities could challenge their competency. It is important to emphasise that all of the students in Uganda were supervised by both Ugandan and UK professionals and were not operating on their own.

Students were able to reflect on this new learning both in terms of clinical skills but also critical thinking. In the following case a prosthetics student suggests that this has contributed to her development as a rounded professional:

This placement was a great opportunity for me to develop my knowledge and develop skills required to become a more rounded and efficient healthcare practitioner. The clinical exposure allowed here in Uganda was almost incomparable to those received in the course so far in the UK. Being in a developing country also increases the amount of critical thinking required to fulfil a job role of an orthotist or prosthetist ... Going during this aspect of our studies has been extremely beneficial in terms of clinical exposure and I have learnt a tremendous amount of information, skills etc. (Prosthetics \& Orthotics, Uganda)

In the final example in this section the nursing student reflects on the value of her learning to her wider professional role. However, she also makes an important point; that the placement enhanced some skills but did not cover all aspects of her 'skills book':

On reflection it has made me a better nurse not because I've seen any wild animal attacks or rare diseases. I didn't work on all of the skills from my skills book but I did work on getting the basics of care right. I realised how important it is to monitor the small changes in patients and how crucial documentation is to understand how the patient is progressing. (Nurse, Uganda) 
In this case, she emphasises the back-to-basics quality of her learning rather than the exotic learning she may have anticipated. Certainly, it is these skills that she will now be utilising in her placements in the UK. Her point about not working on all the skills is important however. It is much more difficult in a low resource setting to ensure a comprehensive skills enhancement in line with any UK curriculum and this has implications in terms of skills mapping and potential augmentation on return. This would certainly be the case if the skills map included working with specific forms of equipment, for example. A number of students explained how the clinical exposure helped develop their self-awareness and shape or confirmed their future career aspirations:

I've learnt more in that month in Uganda than the four months on my placement in the UK. I want to work in adult mental health. (Social Work, Uganda)

It has definitely been beneficial to my course and to what I want to do in future ... It has made me put a specific thing in a bigger picture. We have to look at those things in our course but we've never had to deal with it practically, until we went out there. (Business studies, Uganda)

In our sister book on the learning of professional Volunteers, we noted the contribution that placements made to confidence in using existing skills (Ackers et al. 2017). Most of the students on the EEP placements spoke of their new-found confidence in their clinical skills. The idea of 'deliberative practice' is associated with a particular theory of learning that emphasises the value of repeated engagement with a particular skill or skill set (Ericsson et al. 1993). In our work on the gains associated with professional voluntarism, we noted the gains that NHS professionals experienced through repeated exercise of pre-existing skills (Ackers et al. 2017). This dimension of learning was also in evidence amongst the students on clinical placements in Uganda.

I feel a bit more confident if something happened to be able to actually resuscitate a baby. (Nurse, Uganda)

It's given me an awful lot of confidence. I thought I was quite confident any way but it enhanced my confidence to step forward in the skills I use in the UK. (Nurse, Uganda) 
In the final case the nursing student explains how this had an immediate impact on her professional practice in the UK:

Confidence was the most important thing. I went back to accident and emergency and I was just so much more confident; when emergencies came in I didn't lose my cool, I had a lot more calm after being there because we dealt with lots of emergencies. I wasn't as panicky because I had been in that situation before. (Nurse, Uganda)

\section{Personal Values and the Patient Interface}

We noted above the focus on equality and diversity in the NHS Knowledge and Skills framework and the emphasis of personal values and value systems in the literature on international placements (Brookfield 1995; Elit et al. 2011). For convenience, the following section distinguishes learning directly related to personal values from that concerning wider social values although of course the two are intrinsically related.

The Francis Report (2013) emphasised the importance of care and compassion at all levels of the NHS workforce. This stimulated a drive toward a 'value-based' strategy (Waugh et al. 2014) which has placed the ' 6 Cs'; Care, Compassion, Competence, Communication, Courage and Commitment (NHS 2016) at the heart of the skills enhancement agenda. In addition to the more explicit clinical skills identified above respondents identified a wide range of less tangible skills and knowledge acquired on placements. These included both personal and professional development and again, embodied tacit rather than explicit (or technical) knowledge (Williams 2006).

Through concrete experience augmented through reflection and selfevaluation, students identified care and compassion as important components of their clinical practice. $57 \%$ and $32 \%$ respectively stated that their placement had a very strong or strong impact on their perception of compassion and the importance of empathy. The following comment is typical:

In terms of nursing, it just highlighted to me how important it is to have that relationship with your patient and to just to be reassuring and things like that because there is nothing worse than when you feel like no one really cares about you, when you're ill. (Nurse, India) 
For some students on observational placements in India, this profound experience coupled with their inability to intervene and assist if a patient was in pain caused some anxiety:

You're not used to seeing patients that are like severely in pain, severely distressed and that in itself is just really hard to deal with and you don't, you're never prepared for that because that's just not something that you would see here. And obviously as a nurse you want to look after people and you want to do your best to care for people and make them more comfortable and settled and you feel like you can't do that for them, and you're like, what am I here for? What's the point, what am I doing? You're just making a dent in it you know, you're not making a difference. (Nurse, India)

As we have explained (in Chapter 1) the India placements were not a part of the Ethical Education Placements Model and our experience of them as observation-only placements has lead us to question their impact on student learning and, perhaps more significantly, their compliance with ethical principles. Having said that, students on clinical placements will also experience a sense of disempowerment at the lack of resources and human resource context and the impact this has on their ability to demonstrate care and compassion:

Throughout my three years [at university] everything that they've taught us is all about the patient, caring for the patient and having empathy and sympathy and you know looking after their needs... once you're out there you just see people dying of reasons that you could literally treat them with no problem over here. (Nurse, Uganda)

It touched me a bit because there was a lot of preventable death over there, people just died but if they were over here probably wouldn't have died ... you feel a bit helpless. (Nurse, Uganda)

Many students found being in a different setting challenging and some felt that systems themselves thwarted practice and their ability to provide appropriate levels of care. It is quite different to witness an absolute lack of resources as opposed to an apparent systems failure resulting in inefficient use or abuse of resources. In the following case the nursing student interpreted the situation as a lack of care on the part of Ugandan health workers: 
In some situations it just seemed as if people didn't really care. If someone was in a lot of pain and they were asking for pain medications, they were like: 'No, the [medicines] round isn't until this evening they'll be fine', you know not really caring that much and obviously nursing is a caring profession. (Nurse, Uganda)

For others, experiencing different systems challenged their own world view. Being aware of the conditions and constraints host systems operated in allowed them to place their worldview in context, and realise that practice is framed by context;

There were only two doctors working different shifts, so I think a lot can't be helped. They try to do the best they can but it could easily be much better, there's no dignity or respect. (Nurse, Uganda)

In other cases, students experienced situations that, in themselves, did not reflect resource shortages in any simple sense. In the following case in India, a midwifery student observed a lack of attention to privacy or consent:

Women had no privacy, even in the antenatal there was more than one woman per room and there was no curtain or anything and it was hard to sleep.... and also the fact that they didn't have to consent to anything, the doctors just did it, although they may have consented but we didn't know, it could have been the language or culture, but it's just second nature to do that here. They also took the baby away straight away, just to make sure it was ok, but it was in a different room. So they didn't get much chance to bond, the baby was just left crying. (Midwife, India)

The historical legacy and enduring effects of structural inequality between high and low resource settings and the differences in positionalities of students from high-income countries ${ }^{4}$ and populations in low-income countries means that there can be assumptions of superiority (Elit et al. 2011). Additionally, 'benevolent imperialism' (Huish 2012) and hubris ${ }^{5}$

${ }^{4}$ It is important to point out that many of the students on placement were themselves from diverse backgrounds including students of African or Asian descent.

${ }^{5}$ Defined as dangerous overconfidence. 
(Hanson et al. 2011) can characterise the perceptions and interactions of students:

The main problem really is their culture and their beliefs... I'd say it makes you appreciate people's dignity much more, because over there they don't do that over there, there's no dignity or respect. It makes you more aware of different cultures. (Nurse, Uganda)

Once they had time to reflect on their experiences, some students recalibrated their initial perceptions of staff in host organisations and were able to place their actions in context:

It's mad because I remember thinking 'Oh my god the way they are doing that is so bad; that is terrible' but actually it's not. Now I've come home and thought about it, that's just how they cope, that's how they deal with such low resources and you know those people are still being treated as best as they can be. It's not bad, it's just they are doing what they can with what they've got. But for us, at first it's shocking. (Nurse, India)

Some students referred to what they perceived as a lack of motivation of healthcare workers in their placement setting. ${ }^{6}$ Again, this was something that had been raised with them prior to undertaking placements in Uganda and most were able to place the behaviour of individuals within a particular structural context, recognising that people's apparent motivation would be shaped by extrinsic factors and the way that systems operate:

Motivation wise that's a big issue of course in Uganda, we went to lectures about motivation and absenteeism. That's part of it I'm sure - other reasons as well - but lower motivation because obviously people would be thinking I'm doing the work of more than two or three people and they are not here. Obviously it affects the motivation, they could be overworked which results in them not going to work and it could snowball out of control. (Nurse, Uganda)

${ }^{6}$ Health-worker motivation has formed the basis of much of our project work in Uganda. Students attended a workshop on this in June 2016 involving local health workers and senior managers. The impact of motivation on health-worker behaviour is discussed in Ackers and Ackers-Johnson (2016). 
These examples emphasise the continuing impact of placements and the important learning that happens post return as students reflect upon and make sense of their experience. Harnessing this and facilitating discussion within peer groups optimises the learning of the individuals concerned and enables it to spread to others. It also provides the opportunity to challenge and reduce the risks that students will draw general and perhaps essentialised conclusions from their immersions. Many of the students felt that their experiences abroad made them aware and appreciative of their privilege in the UK, in terms of available resources and the treatment that patients were able to receive:

There's a lot of people suffering in the hospital which is quite difficult because there weren't many beds, it made you appreciate what we have here. (Prosthetics \& Orthotics, Uganda)

Unsurprisingly, the experience of observing or working in a low resource setting encouraged all students to reflect on the role that the UK's National Health Service plays in providing access to health care on a universal basis irrespective of a patient's ability to pay:

It confirmed how privileged our life is and how precious the NHS is. It was heart-breaking to see a young mum of 20 die for the lack of an antibiotic given on time. (Nurse, Uganda)

This regard for the NHS extends to an awareness of the expertise and commitment of NHS staff - whose cadres they are soon to join - giving them confidence in their own career decision making:

I've got a new-found appreciation for our nurses and the hard work they put in and the level of care that we provide. Everyone slags off the NHS ... you have no idea how lucky you are to have been born in this country, receive the level of healthcare that we receive. Halfway across the world it is a very very different story, and they're so grateful for the care they receive. They don't complain in comparison to [the UK] where like everybody loves to moan. (Prosthetics \& Orthotics, Uganda)

The example cited above exemplifies one of the outcomes of placements that we also discussed in our evaluation of professional voluntarism (Ackers and Ackers-Johnson 2016); namely reflection on the attitudes of 
NHS users. The effect that placements in low resource settings have on reinforcing a commitment to care and compassion goes hand-in-hand with a reflection on the lack of appreciation of many patients in the UK and the demands they place on hard-pressed staff:

It was a massive culture shock going to India, but an even bigger one coming home. I remember being on the ward and a patient was shouting at a nurse because she had given him the wrong meal and he was saying how crap the NHS was, telly wasn't working so he was discharging himself etc. And I just remember thinking, you should be thankful, you've got a bed, clean bedding, I didn't say anything but I thought it. (Nurse, India)

It is important that opportunities are given within the curriculum for students returning from placements to make sense of these sometimes conflicting feelings to understand what care and compassion means in different settings and how to handle such patients. In this way, through reflective questioning of both the challenges and assumptions of practices, students demonstrated an increased understanding of different value systems (Brookfield 1995; Elit et al. 2011; Murdoch-Eaton and Green 2011).

The sense of frustration or helplessness that students experience can extend to trauma particularly when students witness deaths. All of the child nurses working on the neonatal units in Fort Portal experienced neonatal deaths and other students experienced maternal deaths. Students were also upset by the very different approaches to death in Uganda. It is important that all parties involved in educational placements in low resource settings are prepared for the level of trauma involved and find ways of managing expectations and experiences of this from induction processes through placement supervision and during their return to study. Perhaps the most important component of this is the provision of supervision by UK professional volunteers on the ground who are able to mentor and support the students. Whilst exposure to death and to cultural differences in the treatment of dead patients can be highly traumatic, this is also an important source of learning for students that they are often insulated from in the UK. It is all too easy, faced with the immediate exposure to death, to judge patients and health workers and find them lacking in care and compassion:

It was horrible. There was a dead baby on the floor one day. But it was kind of good that we were exposed because that can never happen here ... there is no compassion over there; that was one of the hardest things coz when one 
of the babies died, and the doctors saw them dead, there was no grief, no compassion, no emotion, it was just really cold. So it was a learning part for me that I will never do it like that. (Nurse, Uganda)

This case takes us interesting another area of critical learning for students; typically referred to as 'cultural competence'.

\section{Cultural Competence}

The introduction to this book referred to the impact of globalisation on UK universities. The forces of globalisation and internationalisation have and continue to have a massive impact on the National Health Service both as an employer and service provider. Perhaps the most immediate example of this concerns global labour markets in terms of both exporting UK professions and importing their foreign peers. Staff shortages have been commonplace within the UK National Health Service since its creation and it has responded to these through international recruitment. ${ }^{7}$ The NHS has a code of practice on international recruitment from low resource settings which aims to reduce the damaging effects of 'brain drain':

Any international recruitment of healthcare professionals should not prejudice the healthcare systems of developing countries. Healthcare professionals should not be actively recruited from developing countries, unless there is a Government-to-Government agreement to support recruitment activities. ${ }^{8}$

In practice this code seems to have done little to prevent the leakage of large volumes of health workers from low resource settings into the NHS. Whilst these trends are common throughout the world, the UK is one of the biggest importers of foreign health workers. A report by the Organisation for Economic Co-operation and Development (OECD 2015) has shown that $35 \%$ of NHS doctors were born abroad, putting Britain ahead of every

\footnotetext{
${ }^{7}$ There is not scope in this book to comment on the wider ethical dynamics of these processes but for discussion.

${ }^{8}$ http://www.nhsemployers.org/your-workforce/recruit/employer-led-recruit ment/international-recruitment/uk-code-of-practice-for-internationalrecruitment
} 
other country in the European Union except Luxembourg. The same report highlighted a sharp increase in the number of foreign born nurses working in the UK NHS, rising from $15.2 \%$ in $2001 / 2002$ to $21.7 \%$ in $2011 / 2012$. It explains how, within this same decade, $40 \%$ of the overall growth in the number of doctors and $65 \%$ of the growth in the number nurses can be attributed to the arrival of foreign workers. Many of these foreign workers come from low- and middle-income countries; for example in England as at 30 September 2015, there were 18,096 NHS Hospital and Community Health Services staff from India, 479 from Uganda, 4,142 from Zimbabwe, 5,124 from Nigeria and 13,533 from the Philippines amongst many other countries (NHS Digital 2016)

Not only has the composition of the NHS undergone a transformation but wider society has become far more diverse and multicultural than ever before. OECD figures from 2013 estimated that $12.3 \%$ of the UK population were 'foreign born', equating to over 7.8 million people. The differing demographic, socioeconomic and cultural backgrounds lead to differing health needs which must be catered for by the National Health Service. In order for this to happen, staff require additional skill, competency and experience to deal with the less familiar and potentially more complex patient cases. Macfarlane and Dorkenoo (2015) examine the example of female genital mutilation (FGM), estimating that the number of women aged 15-49 with FGM born in countries in which FGM is practised but living in England and Wales had increased from an estimated 66,000 in 2001 to 103,000 in 2011 . They attribute this increase largely to immigration to the UK from African nations where FGM is commonplace such as Somalia, Egypt, Guinea and Djibouti. The rise in cases of FGM has prompted widespread health policy review and staff guidelines and training recommendations (Topping 2015). Other similar cases can be found in terms of the prevalence of infectious diseases, such as HIV and Tuberculosis, for which rates are higher for non-UK-born people. In terms of mental health, Raphaely and O'Moore (2010) concluded that higher rates of depression and anxiety exist among refugees and asylum seekers than the national population or other categories of migrants.

These trends highlight a large and expanding international workforce and a complex, diversifying range of patients within the UK National Health Service. In many ways, professionals from all over the world are working alongside UK health staff to treat patients from all over the world. This leads to greater demands in areas such as workforce integration, 
cultural awareness and sensitivity, competency and human resource management. It also requires staff to have additional skills in the areas of communication and teaching to allow for effective cooperation and the exchange of knowledge, skills and capabilities in the workplace. This is the context within which the development of ethical educational placements is taking place.

In the introduction we questioned whether it is possible for students to unproblematically become 'culturally aware' or develop 'cultural competence' through merely participating in international placements. Practically all of the students mentioned culture, usually in relation to difference, in terms of different systems, ways of working and values. Our survey suggested that $78 \%$ and $18 \%$ of students respectively believed their placement had a very strong or strong impact on their cultural awareness.

Returning to the discussion above about death, some students were able to reflect on their experiences from the perspective of cultural differences rather than concluding simplistically that Ugandans lacked compassion. In the following case, the nursing student recognised the complex relationships between human emotions and outward displays of grief:

The women, when they are having babies and in pain, they just get on with it. There was one woman who was basically just smiling at me the whole way through her labour, she looked so beautiful as well and I just thought oh my god! But then there was one baby who was going to die and when the mum was told she didn't show a massive amount of emotion. I don't know if it's because that's so common to them it was just quite strange to see. (Nurse, Uganda)

Another nursing student describes witnessing the death of a baby in Uganda:

One of the challenges I faced was the lack of emotion people show when they have a baby or if somebody passes away. I found this very challenging especially when somebody passed away. I could not understand how they did not cry. I overcame this by learning that it is part of their culture not to show any emotion and they often do that in private. (Nurse, Uganda)

Some students were positive about the differences they witnessed, demonstrating epistemic humility through highlighting aspects which they felt 
were lacking in the UK. By way of example, many recognised the role played by the extended family and the wider community in supporting patients. This was evident across a number of disciplines and in both the observation-only (India) and hands-on (Uganda) placements. In the first case reported here the paramedic student spoke of the importance of family to patients in Uganda:

I was able to witness how much family assistance with care is relied on, and the generosity of members of the public towards those without families to help their recovery. (Paramedic, Uganda)

Similar comments were made by a midwifery student involved in the India placement who mentioned the support provided by family members reflecting on how this would be handled in the UK:

In India they have the whole family sleeping in the corridor waiting for them. In our hospital we don't even let them wait in the waiting room when their daughter's in labour, they have to do down stairs to Costa ... So the difference is just mad, and like for that woman it could be completely reassuring having mum and dad there. (Midwife, India)

The student in this case is clearly considering some of the potential benefits of having family in the delivery room. She does not reflect at this point on some of the potential risks associated with this in terms of infection prevention or the privacy of other patients; these are issues that could form the basis of interesting discussion about care in context and the boundaries of individual freedom on return to the UK. Others highlighted how family members were kept informed about patients which enabled them to play a meaningful role in supporting their care once discharged from hospital. One of the mental-health students placed in Uganda explains how the family are more engaged in the care of their family members:

I think there was a lot more family involvement in Uganda like in the mental health unit than in the UK [where] you tend to treat the person. In Uganda they connect with the family member to look after them so you got the chance to explain to the family member what condition it is and how it impacts them so that they understand better so I think some of the families in Uganda understood the conditions better than sometimes here because 
they never really get that information from a healthcare professional, so that was good. (Nurse, Uganda)

Social work students on community placements in Uganda were also very positive about the role of the wider community in supporting vulnerable members and reflected on how this approach could be beneficial to the UK:

I did learn a lot, the community projects that are there are something that I haven't seen as much of in the UK, there seems to be a lot more community enterprise there. (Social Worker, Uganda)

The social work student in the next case talks about the 'Goats for Life' project aimed at developing social enterprise to enable families to pull themselves out of poverty. While the project itself is unlikely to take roots in the UK context, his reference to the growth of individualism in the UK is immediately relevant to his future role as a social worker:

One of the projects was the Goats for Life project... So we went to the market and bought 4 or 5 goats and delivered them out to the community. Seeing the wider family network and how that goat can bring them all together was good, because everyone came round and everyone was involved, which was lovely to see. You knew that there was a bond there, and with the child there that had no parents. People would help the child get to school and things and people helped the grandmother out, and I think that's important skills to learn. Right now [in the UK] things are stuck behind doors, and we don't know problems with our own neighbours, and I think it's really important for social workers to learn that community can exist, even if that's in Africa. Were taught not to see it, and that individuality is the trump card, but it is good to see a different way, and see how it can be good to work together. This is a community coming together to help. (Social Worker, Uganda)

In the final case another social work student describes the emphasis on community in Uganda as 'progressive':

I consider the community social work method which I have experienced in Uganda as very progressive, effective and efficient. (Social Worker, Uganda) 
It may come as a shock to many to see social work in Uganda described in such positive terms. One of the social science students similarly described a local midwifery-lead health centre as fitting perfectly with her own personal commitment to natural child birth and rejection of the medicalisation of maternity services in the UK (as she saw it). It would be useful to work through these perceptions with students on return to understand what lies behind them, not as a criticism of Ugandan systems but rather to develop a deeper critique. Community social work, 'natural' childbirth and the involvement of families in mental health may be necessary responses to fundamental gaps in systems; the lack of public services as much as attention to cultural differences. Nevertheless, these observations provide the basis for critical reflection and learning.

Although we question whether just being in a different context builds culturally competence, for some students, having the experience of being in a different setting gave them knowledge of a different country, culture and environment and engender confidence about difference. In the following example a mental-health student suggests that the immersive nature of her experience in Uganda will support her future practice in what is a very multi-cultural environment:

I felt I came back and got more understanding of the different cultures because Liverpool's really diverse; you get a lot of different patients from all around the world and I feel like I can sympathise with them a lot more and understand them a bit more. (Nurse, Uganda)

It is interesting to reflect on this case as the student is deeply embedded in a very diverse home environment; far more diverse in fact that the one she experienced in Uganda. We would argue that it is as much the students' experiences of being an outsider in Uganda or India that hones this experience as it is the exposure to cultural difference per se. The effect of the placements on students' awareness of culture was evident both in the Indian and Ugandan contexts. Echoing previous discussion about the role of family in health care the following student reflects on her placement in India:

It's made me a better nurse because the UK is becoming more multi-cultural so I have to - not that it's a bad thing - I have to face different cultures and to be able to experience their culture and what they normally do. The patients' family come in to wash them, to feed them [in India] whereas here the healthcare systems do that, the nurses do that care. So if they felt 
more comfortable with the family, if it was appropriate, we would encourage the family to come in to offer that care. That's what I would like to do if I was running a ward. (Nurse, India)

Clearly there are many reasons why this approach is both necessary in India and Uganda as these roles are not considered a part of normal health worker roles and would present real challenges to the NHS, in terms of privacy and infection prevention control. Once again experiences such as these and the critical thinking they provoke provide fertile ground for discussion with peers on return rather than immediately transportable policy options.

In the spirit of epistemic humility, having particular concrete experiences and being able to reflect on them and conceptually abstract such experiences, gave some students the opportunity to reconsider how they would practice in the future, in terms of how they would relate to both colleagues and patients:

I wasn't too aware of understanding people and the differences they have due to culture and religions and. I think just having a bit more knowledge ... if I was to obviously care for a patient who was of a different religion or culture I think I would probably take the time to go away and actually learn a bit more about it and to be able to understand them a little bit better like in that sense. (Nurse, Uganda)

The midwifery student in the following case reflects on her experience of observing mothers in India and explains how this has enabled her to understand Indian women's attitudes towards breast feeding in the UK:

We learnt a lot of cultural stuff. Now I can understand why Indian women sometimes act the way they do here. They're not rude, that's just how they are and their culture and they're not used to us being friendly. I've come across a few Indian patients here and I've just accepted this is what they want rather than saying that's just how we do it here. They tend to bottle feed the first 3 days, just because of their culture. Before I would tell them not to now I just accept that's how they are. (Midwife, India)

Once again this raises points for discussion with her tutors and peer group; it may be that from a professional perspective simply accepting bottle feeding is deemed inappropriate or out of line with public health protocols in the UK. Witnessing cultural difference does not necessarily mean accepting all forms of behaviour but it does help to understand what lies 
behind that behaviour. The following example represents similar points for discussion:

To put it into context, I was working on a ward (back home in the UK) and there was an Indian lady having surgery and there was a lot of her family around her bed, like a lot, which you see in India because that's their culture. But I remember a few of the care staff where like 'oh no we can't have that many relatives, oh my god no they've got to go' and it was only because I had been to India and I knew that that's what happens there and that's their culture and it sounds bad but I said to my colleagues it will make it easier for you because they want to look after their relative whilst they are a patient so let them and you just be in the background if they need anything. (Nurse, India)

The nursing student in the next case talks about how the awareness she has gained in India will help her relationships with Indian health workers:

A massive thing I gained in cultural awareness, both for patients and also there are a lot of Indian nurses coming over here and now I feel I have a better relationship with them. To be honest, I used to think they were lazy, because they didn't do personal care but now I know they don't do it and sometimes you'd ask them and they would just look at you and not do it. So now, I just don't ask them now and it's not that they are doing anything wrong it's just how they are taught and it's their culture. And I know you can say they should work within the NMC [Nursing and Midwifery Council] code of conduct but India has given me that awareness of how to deal with that stuff. (Nurse, India)

The students' conclusion that it is OK for nursing students from India not to engage in personal care is quite alarming. Certainly it is essential that foreign staff in the NHS work to the same protocols as their UK peers but understanding these cultural dynamics and global differences within the mixed economy of care is critical to encouraging individual behaviour change.

Finally, a number of students emphasised how the cultural learning they had engaged in was in fact a two-way process. Examples of sharing information, knowledge exchange and developing understanding of how different contexts and systems operate appeared to be key:

I'd say definitely like culture-wise, they gained things because they would ask all sorts of questions about what it's like, like what we think and stuff like 
that, especially our buddie learnt loads about what it's like over here because she was asking us so many questions and stuff... We learnt as much as they did really, because we'd just swap the stories. (Midwife, India)

\section{Communication}

The enhancement of communication skills is identified in much of the existing research on learning outcomes (Jeffrey et al. 2011). In our survey, $40 \%$ and $43 \%$ of students respectively felt that their placement had a very strong or strong impact on their communication skills, stating they were better able to adapt the way they communicated to fit with different contexts and situations. In this way, professional skills development and acquisition (communication) could be understood to link to the development of other skills (managing difficult situations):

I learnt so much not only to help me within my nursing career but I learnt a lot about different cultures and developed my communication skills and my ability to cope under stressful and difficult situations. (Nurse, Uganda)

Some students reported challenges in communicating with staff and patients, but were able to improvise in the way they communicated, demonstrating flexibility and adaptability, and recognised that developing communication skills was important for future practice:

The language barrier was a massive challenge. Most of the patients I came across could not speak English and I could not speak their language. The nurses and healthcare workers also did not speak much English. This made it very difficult when communicating as I often had to use hand gestures. (Nurse, Uganda)

When talking about communication some students demonstrated humility, awareness of privilege and difference and reflected on what it is to be in the minority or perceived as 'other':

You just sort of like get annoyed with those who don't speak English, but we got culturally aware... we were the only ones speaking English, and you were just more aware of how they must feel when they are somewhere [where nobody speaks their language]. (Nurse, Uganda) 
The following podiatry student talks about her skills in non-verbal communication in Uganda:

Obviously not everyone over there speaks English but you can still kind of communicate, with expressions in the way you behave. Sometimes you didn't know what they were saying but you could still get an idea of what they were saying by their body language and expression. (Podiatrist, Uganda)

Even where staff were speaking English the following nursing students showed an awareness of how miscommunication can arise:

I haven't worked with any African patients since being in Uganda but I think I probably have more of an understanding of accepting, if you ask them to do something or you know the way they reply, I think I've had more patience and I know why they're saying what they are, and just try and approach the situation a bit differently but not be upset or angry at the fact they've turned around and said something completely out of order. (Nurse, Uganda)

In the following case the student nurse learns how to interpret a use of English in Uganda that she is unaccustomed to and accept this as a facet of cultural differences; her own language suggests that she continues to view this as 'wrong':

At first you get on with it, like trying cursing under your breath but then you think well sometimes it's different cultures and the way they deal with things... sometimes people don't necessarily mean to be rude or awful, it's just how they've spoken most of their life or how they speak to people, and nobody's ever really turned around and said: you shouldn't say that to people. (Nurse, Uganda)

Other students recognised the need to modify and adapt the way they communicated to better relate to Ugandan patients. This is of particular importance in the field of mental health:

I think it's really good ... the cultural aspect of it, the communication. We used too much clinical language with the patient so they taught us how to speak to people. It was good like that. (Nurse, India) 
The development of communication skills amongst students was also remarked upon by programme leads in the Universities and by Professional Volunteers:

There was a big development of confidence, and being in a situation where people don't always speak the same language as you, or choose not to in some cases. I think it is using your communication skills in a different environment. People have mentioned things about their self-awareness of their inability to communicate... They learn more of the softer skills, like communication and working in a team and they learnt to assert themselves sometimes and come across in a certain way. (MMU University Programme Lead)

They have really come together as a team and they are very good at supporting each other, and they have learnt to communicate with staff... it all links to the team working. (PV, Uganda)

\section{Teaching and Presentation Skills}

As we noted in Chapter 2 students undertaking placements in Uganda are given a range of potential teaching opportunities. These included contributing to teaching on the $\mathrm{K} 4 \mathrm{C}$ sponsored degree programme in midwifery alongside our professional volunteers. They also had the opportunity to join local MMU students in social work and community development workshops and business students worked directly on joint project work as part of the hand hygiene project. This latter project involved some teaching in in local schools on basic infection prevention control. Perhaps less planned, EEP students found themselves working alongside quite large volumes of students from local colleges left to find their way without supervision in Ugandan health facilities. A number of students indicated that their placement provided them with the opportunity for personal growth and professional development, and developing teaching and presentation skills were key areas where this seemed to operate. It is apparent that such opportunities were felt to benefit future career prospects in terms of enhancing CVs, but students were also keen to make a contribution to the host setting. Some students were initially reluctant to put themselves forward, but were able to reflect on how the (concrete) experience helped their skills development. This 'learning through doing' occurred across a range of disciplines and student cohorts but was limited to those students who were involved in 
practice-based placements in Uganda. The respondent in the first case describes her initial reluctance to get involved in teaching:

We didn't want to do it because we hadn't done it before and at first the students laughed at some people and it ran over quite a long time but we had chance to talk to them and have a good discussion. It also helped our presentation and teaching skills. (Social work)

In another example, paramedic students were invited to develop emergency first aid training for local ambulance drivers in Uganda who receive no training at all in this area and are not accompanied on their journeys by health professionals. In this case the students jumped at the opportunity without any reservation:

The training we did came off well, some of the nurses came and the ambulance drivers and we were very pleased with how quickly the skills were coming on; we taught CPR and how to dress wounds and deliver babies. I think we helped a lot there because they said they do have to do a lot in the community but don't really have the training for it and I think we've been a bit of an impact there. (Paramedic, Uganda)

The prosthetic and orthotic students were given opportunities to work alongside Ugandan students in co-mentoring roles. In addition, they took part in some large group teaching:

The opportunity arose for me to conduct an anatomy lecture discussing upper limb bony anatomy, musculature, as well as blood and nerve supply to the second year students studying orthopaedic technology. I have never had the same number of students listening to a lecture before and hence it was a great experience for me to increase confidence, reinforce my ideal subject area etc. (Prosthetics \& Orthotics, Uganda)

This experience had an important confidence-building effect enabling the student to reflect positively on her own knowledge and skills base; there is nothing like teaching to make you aware of how much you know! We have already pointed to the importance of 'back-to-basics' learning in low resource settings. Students' awareness of the neglect of some of these foundational systems and skills encouraged them to engage in teaching in this area: 
With a few people from my group we also did a short teaching session at Mountains of the Moon University to Nursing and Midwifery students on the importance of regular clinical observations and noticing signs of deterioration, the use of observation charts and the introduction of an Early Warning Score system. The session was quite successful and the students seemed really interested and willing to learn and change how they do things. (Nurse, Uganda)

The engagement in teaching encouraged students to see that their roles as health workers were more than simply service delivery; teaching is a core if often invisible component of everyday practice. And being aware of this supports attention to their own position as role models for other health workers:

Up until my last module at university, I never considered myself to be someone that would be seen as a teacher, yet working in Uganda made me see that as a nurse we continue to learn and teach every single day. Whether it is showing someone a small task or helping to better someone's performance of different skills. (Nurse, Uganda)

In the final case cited here a business studies student became involved in teaching large groups of students in Ugandan schools about hand hygiene:

I'll be more confidence in public speaking I won't be so shy now and I will feel more comfortable approaching complete strangers as this was challenging from the beginning but then it started to feel more normal. (Business, Uganda)

Student responses to the EEP survey were more varied regarding the impact that their placement had on their teaching and presentational skills. Fifty four percent believed their placement had a strong or very strong impact, $32 \%$ believed their placement had a moderate impact and $21 \%$ believed their placement had little or no impact. The reason for this variation is likely to be that not all students had the opportunity of engaging in university teaching or conducting presentations. The post-placement qualitative interviews however suggested students that did engage in these activities believed them to be highly beneficial to their learning.

The final section here concerns learning associated with leadership. 'Service improvement' is one of the areas identified in the NHS Knowledge and Skills framework. Service improvement is broader than 
leadership and may imply personal skills and values as well as influencing those of others.

\section{Service Improvement and LeAdership Skills}

As indicated above, Williams and Balatz (2008) identify management 'know-how' as a form of knowledge and management and leadership skills formed perhaps the most important element of learning amongst professional volunteers (Ackers et al. 2017). It is perhaps unsurprising that fewer students referred explicitly to management skills as something they had acquired or developed while on placement. This could reflect the early stage they were at in terms of their career, although some students were able to think ahead to how such skills could be deployed in the future. In the first example the nursing student felt empowered by her teaching role in Uganda reporting gains in confidence and communication:

Some of the health professionals over there wanted to learn from us. A lot of it was about delegation and was teaching there, and it's quite useful coming back to the UK because it's made me a lot more confident really. Better communication, able to do management really of a ward which is what I was expecting. (Nurse, Uganda)

In the next case the nursing student suggests that the placement increased his ability to exercise autonomy:

I think leadership and management is quite a big thing in nursing, and being able to manage myself in Uganda has helped massively. I went out there with an open mind. It's made me realise how lucky we are over here, and how well people can do with limited resources.... You are supposed to work with autonomy in nursing, and I can do that more now. (Nurse, Uganda)

Whilst relatively few students singled out management as one of the key learning outcomes, we would argue that management or leadership are composite qualities which depend heavily on other core skills embracing all of the skills discussed above. Survey responses for leadership and teamwork were very positive, with $73 \%$ of students believing their placement had a strong or very strong impact, $19 \%$ a moderate impact and $8 \%$ little impact. Responses regarding 'management skills' were weaker but still 
relatively positive with $59 \%$ stating a strong or very strong impact, $23 \%$ stating moderate impact and $18 \%$ stating little or no impact.

\section{Personal Growth}

We started this chapter with a series of quotes from students indicating the transformational quality of learning on their placements. In this final section, we return to this but in the context of what can best be described as personal growth. In many cases this was not all about new learning but about increased self-awareness and confidence at having been put to the test, immersed in an environment completely outside their previous experience and comfort zone. The EEP survey revealed that $48 \%$ of students believed their placement had a strong or very strong impact on their personal commitment and motivation, $12 \%$ stated a moderate impact and only $5 \%$ stated little or no impact. The following comment is quite typical and shows the effect of the placement in terms of what we have called 'mobility capital' increasing enthusiasm for and confidence in future mobility:

This trip was extremely relevant to my degree. It has not only benefitted me as a mental health nurse but raised my confidence as an individual. It has inspired me to challenge myself in working outside the UK later in life. (Nurse, Uganda)

Many students spoke proactively of the impact of the placements on their future plans and were actively considering future stays in low resource settings:

Personally, I had an absolutely brilliant time. Being a part of this Ugandan placement has only strengthened my desire to continue studies and aid research/projects that can help communities. (Prosthetics \& Orthotics, Uganda)

It is an experience that will remain with me for the rest of my life and it has confirmed my desire to go back to Africa to work in similar conditions. (Nurse, Uganda)

We referred in Chapter 1 to the fact that most if not all of the students who travelled to Uganda and India with the benefit of bursaries would not, otherwise, have had access to this kind of opportunity either for purely financial reasons or because they lacked the confidence to try to 
access placements. This was evident in the way many students talked about the experience of living in a foreign country with other students and away from their families for the first time:

It's made me a different person in a sense of, because it was my first time away on my own without my family it's made me more independent, it's made me appreciate life more. (Nurse, India)

It made you stronger as a person. It got quite emotional at times and I think being away from home as well, I've been away from home before but not for a month in a country that's completely different. Also being away from parents and family and having to make relationships with people you don't know, well you have met everyone once before but you were in that environment for 4 weeks and you kind of had to just get on. (Nurse, India)

Many students spoke of the personal journeys they had faced associated not solely with clinical exposures but with living amongst other students and outside of their comfort zones:

It was totally different, it was like out of your comfort zone... I was like doing things I wouldn't normally do. Like even living with a load of people that you didn't know, you've never lived with before... I didn't think I'd cope well but I coped a lot better than I thought I would. (Nurse, Uganda)

Well I've learned I'm even more emotional than what I think I've got, but then at the same time I'm a lot stronger than I thought I was. (Nurse, Uganda)

My life has changed quite a lot since I've been back such as I am moving out because I feel more independent and I came back thinking maybe I should be doing more things such as going for more job opportunities and making the most of things. (Social Worker, Uganda)

It was the worst and best thing I've ever done in my life that's all I can say. Worst in terms of it was hard, especially for me I'm just not used to that... but the best in terms of learning about culture, health care in a different world and about myself. (Nurse, India)

It has just made me more confident, and I now know I'd be able to go elsewhere confidently... Definitely, it has helped with my confidence. I had an interview when I got back which I got and there were only two places so I was very pleased to get it... I think going to Uganda made me more confident because you get thrown into different situations I have to get on with it. (Social Worker, Uganda) 
I have learnt that I am a very strong person, I can do anything I put my mind to and it has also given me a lot more confidence as a student nurse, that I can carry forward to when I am qualified. (Nurse, Uganda)

Even just outside the placements spending a bit of time in a different country increases your confidence... not sure how I'll translate it into practice but I learnt a lot about being in difficult situations and how to handle them (laughs) and a lot about emotional intelligence and how to deal with conflict and things like that and how to manage like stress as well. (Occupational Therapist, Uganda)

When faced with the situations and experiences I did in Fort Portal, Uganda, I bloomed and I know for a fact that not only has it made me a better professional but that it has changed my life forever and will ultimately impact on my career and future planning of it. If I had the chance to go back and do it all over again I wouldn't change a thing. (Nurse, Uganda)

\section{EMPLOYABILITY}

Although a number of students expressed concern even at being asked about the impact of their placement on their future employability, perhaps sensing that this was unethical and sounded 'selfish' it was clear that students recognised the potential career-enhancing effects of the placement experience in terms of 'giving them the edge' or 'setting them apart'. Responses to the survey were overwhelmingly positive with $95 \%$ of students believing their placement was very beneficial for their future career and employability. This applied equally to students on observational placements. The following comments are typical:

It will show that I've got a determination expand my horizons and gain further experiences and take on challenges. (Nurse, India)

I never really thought 'this can go in my portfolio' and 'this can be good for jobs' but everyone's telling me, even on my last (UK) placement, 'that'll be really good for interviews and jobs'. (Physiotherapist, Uganda.

I think It puts me in a very strong position because in university they say all the time when it comes to job applications; have you done something that would make you stand out from the crowd...that automatically gives you a win in the interviews - you've got a really positive experience you can talk about all day - that makes you much more employable. (Nurse, Uganda) 
It will give an edge. Not a lot of nurses do it so to do it as a student shows you can manage in a different setting. You can manage in scarce resources. You can work on your own, know your limits, you're resourceful. There's loads of indications to an employer that you can work under stressful situations with new encounters and work through it in a logical way to get the most out of it for your patient. So just by saying that you've done this to an employer shows you have all these qualities and shows that you will go that extra mile to go and make a difference and improve patient care. (Nurse, Uganda)

\section{SUMMARY}

This chapter has summarised the findings of the survey and qualitative interviews to demonstrate the impact that educational placements have on student learning. The results show that the learning outcomes are profound and directly relevant to undergraduate curricular and NHS objectives. There was an overwhelming belief that the placements contributed to their future skills and employability. Unsurprisingly clinical skills learning was much more pronounced amongst students undertaking the EEP placement and experiencing hands-on clinical work under the direct supervision by Professional Volunteers and Ugandan health workers.

Open Access This chapter is licensed under the terms of the Creative Commons Attribution 4.0 International License (http://creativecommons.org/licenses/ by $/ 4.0 /$ ), which permits use, sharing, adaptation, distribution and reproduction in any medium or format, as long as you give appropriate credit to the original author(s) and the source, provide a link to the Creative Commons license and indicate if changes were made.

The images or other third party material in this chapter are included in the book's Creative Commons license, unless indicated otherwise in a credit line to the material. If material is not included in the book's Creative Commons license and your intended use is not permitted by statutory regulation or exceeds the permitted use, you will need to obtain permission directly from the copyright holder.

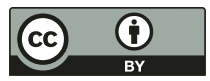

